\title{
TENDENCIA DE LA TEMPERATURA EN UNA PLANICIE DE MAREA DEL ESTUARIO DE BAHÍA BLANCA (PERÍODO 2000-2007)1
}

\author{
Débora Beigt ${ }^{a}$ y M. Cintia Píccolo ${ }^{a, b}$ \\ ${ }^{a}$ Instituto Argentino de Oceanografía, Bahía Blanca, Argentina

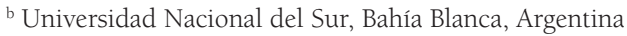

\section{RESUMEN}

Se analizó la tendencia y comportamiento de la temperatura en una planicie de marea del estuario de Bahía Blanca (Argentina) para el período 2000-2007. Mediante termistores se registró la temperatura del aire, agua y sedimento cada 10 minutos. Se observó una tendencia de aumento en las temperaturas

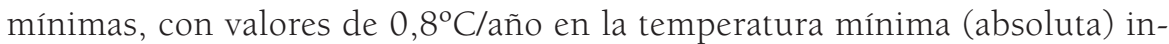

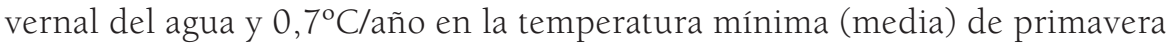
del aire. Las máximas medias estivales del aire y del sedimento mostraron una tendencia negativa, con un descenso de $0,5^{\circ} \mathrm{C} /$ año. El análisis espectral de las temperaturas en el estuario presentó evidencias de periodicidad interanual ( 2 - 6 años) en el aire, agua y sedimento, mostrando un patrón de variabilidad estrechamente relacionado con las periodicidades típicas del fenómeno ENOS (2 - 7 años). El análisis de correlación entre las anomalías térmicas y el IOS indicó que las temperaturas del aire, agua y sedimento en el estuario son sustancialmente influenciadas por el forzamiento remoto. La ocurrencia de eventos El Niño coincidió con temperaturas del aire y del agua superiores a los valores medios en el estuario, y viceversa durante los eventos La Niña.

1 Este trabajo corresponde a proyectos financiados por el Consejo Nacional de Investigaciones Científicas y Técnicas (CONICET) (PIP 2158) y la Agencia Nacional de Promoción Científica y Tecnológica (ANPCYT) (PICT 07-12421). Investigador principal: M. Cintia Piccolo,piccolo@criba.edu.ar 
Palabras clave: tendencia - temperatura - estuario de Bahía Blanca

\begin{abstract}
Temperature trends in a tidal flat of the Bahía Blanca estuary (Argentina) were analyzed during the period 2000 - 2007. Air, water and sediment temperatures were recorded every 10 minutes by a thermistor chain. Minimum temperatures showed an increasing trend, reaching $0,8^{\circ} \mathrm{C} /$ year (absolute minimum water temperature in winter) and $0,7^{\circ} \mathrm{C}$ year (average minimum air temperature in spring). Average maximum summer temperatures at the air and sediment showed a negative trend of $-0.5^{\circ} \mathrm{C} /$ year. Spectral analysis of the estuarine temperatures evidenced an interannual periodicity $(2-6$ year $)$ at the air, water and sediment, showing a variability pattern associated to the typical periodicities of the ENSO ( $2-7$ years). Correlation analysis among thermal anomalies and SOI suggested that air, water and sediment temperatures in the estuary are substantially influenced by remote forcing. The occurrence of El Niño events was coincident with higher than average air and water temperatures and vice versa during La Niña events.
\end{abstract}

Keywords: temperature trends - Bahía Blanca estuary

\title{
1. INTRODUCCIÓN
}

Durante el siglo XX, y especialmente durante los últimos 30 años de este siglo, se ha registrado un calentamiento climático importante a nivel global (IPCC, $2001 a, b)$. Aunque las variaciones térmicas no son uniformes y se han señalado diferencias significativas entre distintas regiones (Houghton et al., 2001), la tendencia lineal de la temperatura de la Tierra en los últimos 50 años muestra un incremento promedio de aproximadamente $0,13^{\circ} \mathrm{C}$ por década (IPCC, 2007). El Panel Intergubernamental sobre Cambio Climático (2001c) señala, además, que el calentamiento es más intenso en latitudes medias y que el parámetro más sensible a dicho calentamiento es la temperatura mínima. Los patrones recientes de cambios en la temperatura a escala local y regional han sido relacionados con las diferentes fases de las oscilaciones oceánico-atmosféricas (Leathers et al., 1991; Trenberth y Hurrell, 1994; Hurrell, 1995, 1996; Zhang et al., 1997; Enfield y Mestas-Nuñez, 1999; Yasunaka y Hanawa, 2002; Gong y Ho, 2004; Salinger, 2005). Entre ellas, la causa natural más importante de variabilidad climática a escalas de tiempo estacional a decadario es el fenómeno ENOS (El Niño-Oscilación del Sur) (Limsakul y Goes, 2008). Al respecto, Torrence y Compo (1998) estudiaron la serie de tiempo correspondiente a la temperatura superficial del mar en la región Niño 3 y observaron un incremento en la potencia de la señal 
en el intervalo 2-8 años en el período 1960-1990. Labat et al. (2005), por su parte, analizaron la variación del IOS (Indice de Oscilación del Sur) desde el año 1870 y hallaron una fuerte componente interanual (2-7 años) en los períodos $1870-1920$ y $1965-2000$.

Debido a que estos patrones de circulación juegan un rol clave en los patrones climáticos regionales, existe una necesidad creciente de comprender cómo afectan a los ecosistemas a escala local y regional. Numerosos artículos estudian la tendencia y variación temporal de la temperatura en aguas costeras (Koutsikopoulos et al., 1998; Rock et al., 2002; Younes et al., 2003; Nezlin et al., 2004; Preston, 2004; MacKenzie y Schiedek, 2007; Masson y Cummins, 2007; VargasYáñez et al., 2007). Preston (2004) analizó datos de temperatura del agua registrados entre los años 1949 y 2002 en el estuario de Chesapeake Bay (EEUU), hallando un incremento de 0,16 y $0,21^{\circ} \mathrm{C}$ por década en el agua superficial y subsuperficial, respectivamente. El calentamiento se produjo especialmente durante el invierno y la primavera. El autor afirma que el calentamiento continuado del estuario tendrá implicaciones importantes en la estructura y función del ecosistema, así como en el manejo de problemáticas ya existentes, como la eutroficación y la hipoxia béntica (Preston, 2004). En la Bahía Narragansett (EEUU) se estudió la temperatura del agua durante el período 1960 - 1990, observándose un incremento de $3^{\circ} \mathrm{C}$ durante la estación invernal (Rock et al., 2002). Los autores estiman que tal cambio en la temperatura invernal probablemente ha alterado las cadenas tróficas de aguas costeras en el Sur de Nueva Inglaterra e influído en la declinación de peces bénticos, comercialmente importantes en dicha área. Masson y Cummins (2007) hallaron una tendencia de incremento de 0,024 ${ }^{\circ} \mathrm{C} /$ año en la temperatura del agua del Estrecho de Georgia (Canadá) a lo largo del período 1970 - 2005. Nezlin et al. (2004) estudiaron la variación interanual de la temperatura superficial del mar en la Bahía Santa Mónica (California) en el período 1987 - 1997 y hallaron una alta correlación entre dicho parámetro y varios factores de forzamiento externo (esfuerzo del viento, temperatura del aire e índice Niño 3), evidenciando que las variaciones térmicas están reguladas tanto por procesos locales como por forzamiento remoto (ciclo ENOS).

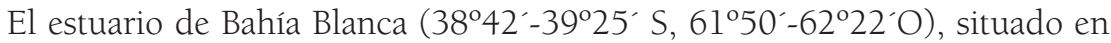
el Sudoeste de la provincia de Buenos Aires (Fig. 1), es un estuario de planicie costera mesomareal. Con una superficie de $2300 \mathrm{~km}^{2}$, es el estuario más grande de Argentina luego del estuario del Río de la Plata. Se enmarca en un clima de transición entre el templado - húmedo del Este de la provincia de Buenos Aires y el templado - árido de la Patagonia (Capelli de Steffens y Campo de Ferreras, 1994). La temperatura media anual en la región es de $15^{\circ} \mathrm{C}$, con una amplitud media anual de $13,7{ }^{\circ} \mathrm{C}$ (período 1951 - 2000). Dominan el patrón climáti- 
co regional los vientos provenientes de los anticiclones semipermanentes del Atlántico Sur (aire cálido y húmedo) y del Pacífico Sur (vientos fríos y secos). Siguiendo a Ángeles (2001), el área del estuario de Bahía Blanca presenta un tipo climático semiárido templado, con períodos húmedos en verano, primavera y otoño (donde se concentra el $89 \%$ de las precipitaciones) y un período seco en invierno. Piccolo y Diez (2004) estudiaron la temperatura del aire en el estuario (estación meterológica de Puerto Rosales, Fig. 1) para el período 1999 - 2003 , hallando una media anual de $15,6^{\circ} \mathrm{C}$, con una máxima de $37,9^{\circ} \mathrm{C}$ y una mínima de $-3,1^{\circ} \mathrm{C}$. Arango (1985) estudió la temperatura media superficial del agua durante el período 1967 - 1984 en diferentes estaciones localizadas a lo largo del Canal Principal (Fig.1). Halló un valor medio anual de $14.7^{\circ} \mathrm{C}$, variando de $21,6^{\circ} \mathrm{C}$ en verano a $8,5^{\circ} \mathrm{C}$ en invierno. En Puerto Cuatreros la temperatura media anual del agua fue $15,2^{\circ} \mathrm{C}$. Piccolo (1987) registró la temperatura del agua de Ingeniero White (Fig. 1) en forma contínua durante el período 1979 - 1985 , hallando un valor medio anual de $15^{\circ} \mathrm{C}$.

Piccolo y Dávila (1991) estudiaron la temperatura del sedimento en una planicie mareal de Puerto Ingeniero White. Un análisis espectral mostró máximos de energía correspondientes a 8 y 3 días y a 24, 12, 8 y 6 hs, indicando la importancia de procesos sinópticos, diurnos, semidiurnos y mareales. Por su parte, Beigt et al. (2003) hallaron las mayores fluctuaciones térmicas en los $15 \mathrm{~cm}$ superiores de la columna de sedimentos, donde se desarrollaron importantes gradientes verticales $\left(0,82^{\circ} \mathrm{C} \mathrm{cm}^{-1}\right)$, especialmente a mediodía y primeras horas de la tarde, con la exposición de las planicies a la radiación solar. El presente artículo analiza la tendencia y comportamiento de la temperatura del aire, agua y sedimento, en una planicie de marea del estuario de Bahía Blanca, con el propósito de identificar sus patrones temporales dominantes y establecer su posible conexión con las diferentes fases del fenómeno ENOS. El estudio se considera una contribución importante para el área de estudio, dado que, por primera vez en el estuario de Bahía Blanca, se analiza una serie de tiempo de 7 años de datos de temperatura registrados de manera contínua a intervalos de tiempo pequeños.

\section{MÉTODO DE TRABAJO}

Durante el período 2000 - 2007 se efectuó una medición contínua de las temperaturas en una planicie de marea ubicada en Puerto Cuatreros (Fig. 1), como parte de un programa de monitoreo de humedales en el estuario de Bahía Blanca. El área se halla en la cabecera del cuerpo de agua. La medición se realizó con un intervalo de muestreo de 10 minutos mediante una cadena de termistores. Se registró la temperatura del aire a $3 \mathrm{~m}$ de altura sobre la planicie, la temperatura del agua a -1 m en bajamar, con un sensor instalado en el muelle de Puerto 
Cuatreros y la temperatura del sedimento de la planicie a $5 \mathrm{~cm}$ de profundidad. Se utilizaron técnicas estadísticas estándar para el análisis de las temperaturas medias, mínimas y máximas. Se estudió la tendencia de la temperatura mediante regresión lineal, cuya significación se determinó a partir de la tabla ANOVA (estadístico F).

Figura 1. Ubicación del área de estudio

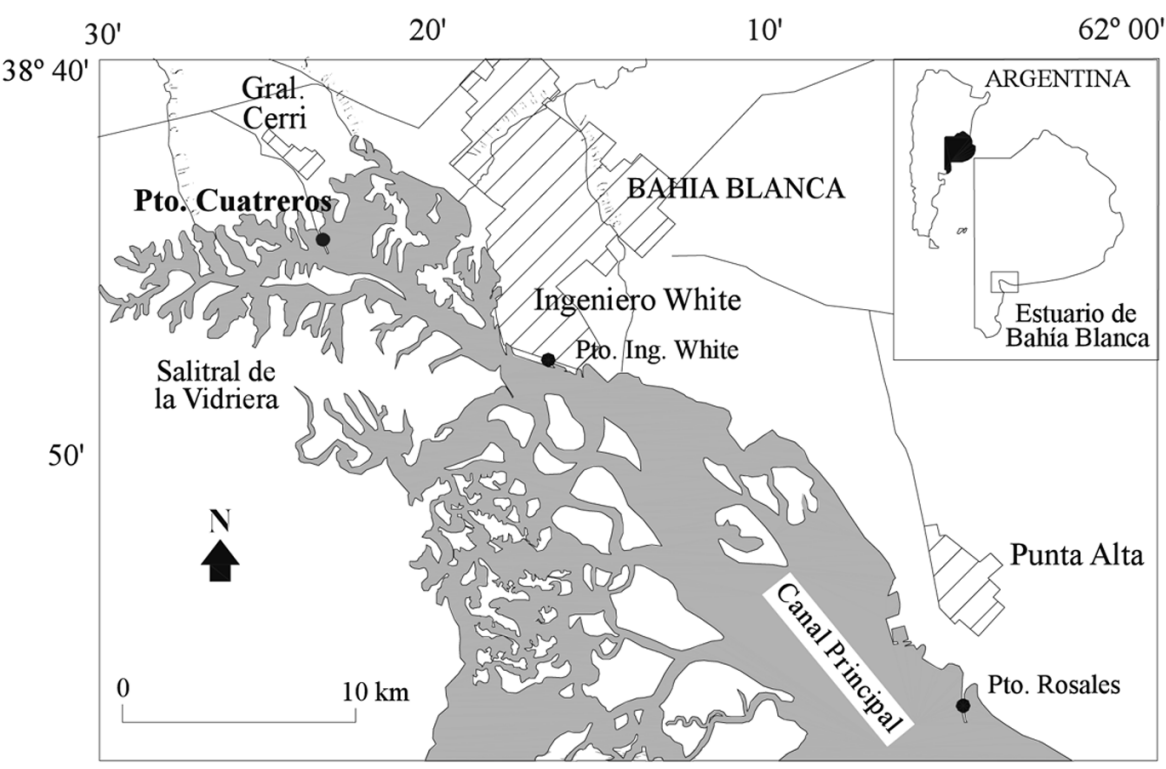

Posteriormente, las series de tiempo fueron convertidas en anomalías desestacionalizadas mediante el ajuste de una función armónica con un período de 12 meses (1) (Nezlin et al., 2004) a los datos de temperatura diaria:

$$
y=m-k \cos ((t-\phi) 2 \pi / 365)
$$

donde: $y$ es la temperatura, $t$ es el día juliano, $m$ es la temperatura media, $k$ es la amplitud del ciclo estacional y $-f$ es el día juliano en que ocurre el mínimo estacional.

A partir de dicho ajuste se calculó el ciclo anual medio, que se removió de la serie de tiempo observada para obtener las anomalías térmicas. Las temperaturas medias diarias y las anomalías se analizaron utilizando la Transformada Rápida de Fourier. Las anomalías en el aire, agua y sedimento se correlacionaron mediante el índice de Pearson y se suavizaron mediante un filtro con medias móviles de 100 días. Estas anomalías filtradas se correlacionaron a su vez con el Índice de Oscilación del Sur (IOS) para identificar una posible influencia del fenómeno ENOS sobre el comportamiento de la temperatura en el estuario de Bahía Blanca. 


\section{RESULTADOS}

\subsection{Temperatura del aire}

La temperatura del aire presentó valores medios diarios de $20,5^{\circ} \mathrm{C}$ (temperaturas máximas), $14,5^{\circ} \mathrm{C}$ (temperaturas medias) y $8,7^{\circ} \mathrm{C}$ (temperaturas mínimas). En tanto, la dispersión o variabilidad fue mayor en las temperaturas máximas (con un desvío estándar de $\pm 8,3^{\circ} \mathrm{C}$ ) y menor en las mínimas (desvío estándar $= \pm 6,5^{\circ} \mathrm{C}$ ) (Fig. 2). Respecto a la tendencia lineal de la temperatura del aire durante el período de estudio, se halló una regresión significativa para las temperaturas mínimas de primavera ( $\left.p<0,05 ; R^{2}=0,591\right)$, las mínimas estivales $(p<0,1$; $\left.\mathrm{R}^{2}=0,475\right)$ y las máximas estivales $\left(\mathrm{p}<0,1 ; \mathrm{R}^{2}=0,485\right)$ (Fig. 3a). Las rectas de regresión mostraron una pendiente positiva en los dos primeros casos, con incrementos térmicos de $0,7^{\circ} \mathrm{C} / a n ̃ o$ y $0,4^{\circ} \mathrm{C} /$ año en la temperatura mínima de primavera y verano, respectivamente. Las máximas estivales, en cambio, mostraron una disminución térmica de $0,5^{\circ} \mathrm{C} /$ año.

El análisis espectral de las temperaturas diarias (Fig. 4) mostró el mayor pico de energía asociado a las señales anuales. En la escala interanual se observaron dos picos, uno de mayor energía a los 3,7 años y otro menor a los 1,9 años. Para atenuar la periodicidad anual que impide una mejor visualización de las restantes fluctuaciones térmicas, se removió el ciclo medio anual (Fig. 5) de la serie original y se calcularon las anomalías térmicas. El ciclo medio anual de temperatura del aire (Fig. 5a) muestra una amplitud térmica de $18,3^{\circ} \mathrm{C}$, variando entre $23,9^{\circ} \mathrm{C}$ a mediados de enero y $5,6^{\circ} \mathrm{C}$ a mediados de julio. Las anomalías térmicas en el aire presentaron correlaciones muy significativas $(\mathrm{a}=0,01 ; \mathrm{p}<0,0001)$ con las anomalías en el sedimento $(r=0,6)$ y en el agua $(r=0,49)$ (Fig. 6). Los espectros de Fourier de las anomalías (Fig. 7) muestran picos de energía en períodos anuales y semianuales (2 ciclos/ año). Con la atenuación de la periodicidad anual, se observan ciclos de 85, 68 días y 44 días con densidades espectrales similares a los ciclos semianuales. Respecto a la variabilidad de más largo plazo en la temperatura del aire, la energía muestra un máximo a los 585 días ( 1,6 años), con otro pico a los 5,6 años.

Las anomalías térmicas filtradas con medias móviles de 100 días (Fig. 8a) muestran valores máximos en el aire a mediados de abril de $2001\left(+4^{\circ} \mathrm{C}\right)$, a fines de marzo de $2006\left(+3,7^{\circ} \mathrm{C}\right)$ y a fines de octubre de $2000\left(-3,4^{\circ} \mathrm{C}\right)$. Las anomalías fueron predominantemente positivas desde agosto de 2002 hasta enero de 2007, presentándose previamente dos períodos de anomalías negativas; el primero abarcó todo el año 2000 y el segundo el período julio 2001 - agosto 2002. 
Figura 2. Valores diarios medios, máximos, mínimos y dispersión de (a) temperatura del aire, (b) temperatura del agua y (c) temperatura del sedimento Período $2000-2007$

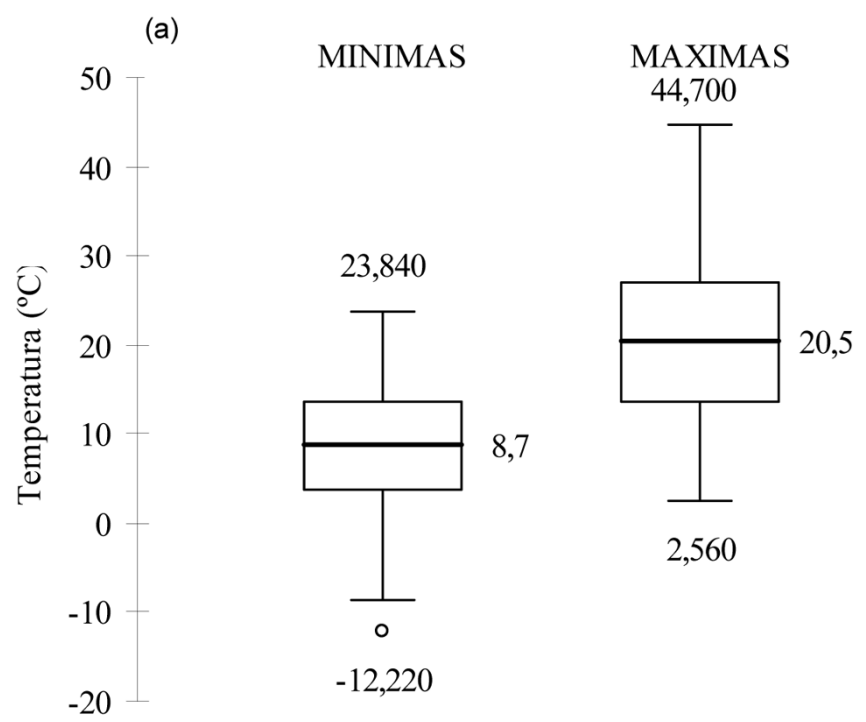

\section{MEDIAS}

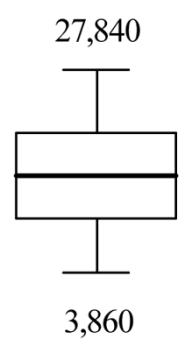

14,5

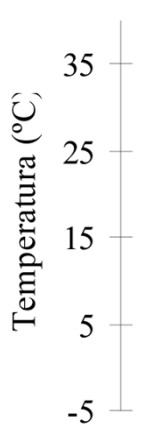

\section{6,380}

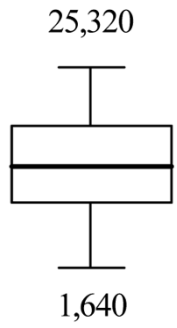

13,6

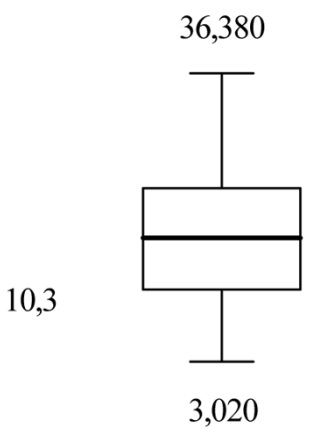

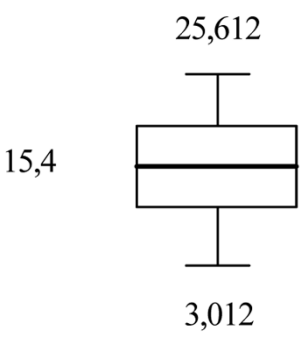

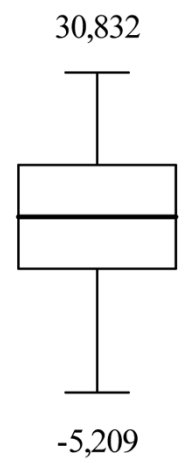

14,5

17,3

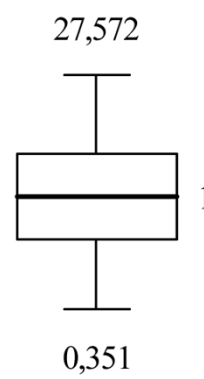


Los valores mensuales del Índice de Oscilación del Sur (IOS) para el período en estudio (Australian Bureau of Meteorology, 2008) se muestran en la figura 8b. El IOS mostró valores predominantemente positivos hasta marzo de 2001, relacionados con el evento La Niña, que comenzó hacia mediados de 1998 y se extendió hasta principios de 2001 (OMM, 2006). En este período las anomalías térmicas en el aire fueron predominantemente negativas. Las mayores anomalías térmicas positivas registradas en el aire se produjeron luego de este evento La Niña. Desde marzo de 2002 hasta mayo de 2005, a excepción de algunos meses, el IOS fue mayormente negativo, coincidiendo aproximadamente con un período de anomalías positivas en la temperatura del aire en el estuario. Cabe aclarar que en 2002 / 03 y 2004 se registraron episodios El Niño en el Pacífico tropical (Poveda et al., 2006). En tanto, durante gran parte del año 2005 se dieron condiciones neutrales (OMM, 2006), registrándose simultáneamente anomalías predominantemente positivas en el aire. En 2006 / 07 tuvo lugar otro evento El Niño (IOS negativo) y las anomalías en la temperatura del aire mostraron valores positivos hasta enero de 2007, cuando las anomalías de la temperatura superficial en el Pacífico ecuatorial comenzaban a debilitarse luego de la ocurrencia de un Niño débil (INTA, 2007).

Figura 3. Análisis de regresión de la temperatura máxima estival del (a) aire y (b) sedimento. Período $2000-2007$

(a)

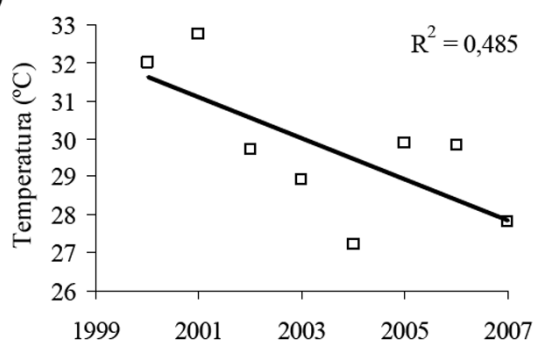

(b)

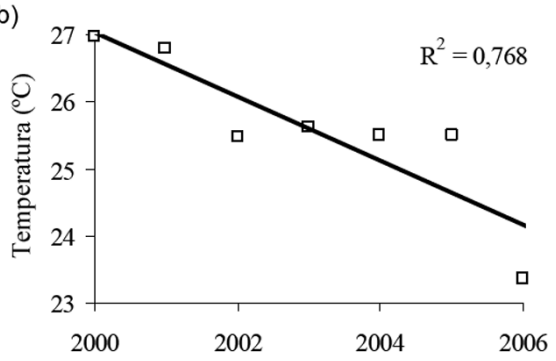

\subsection{Temperatura del agua}

Las temperaturas máximas, medias y mínimas diarias del agua mostraron valores medios de $15,4^{\circ} \mathrm{C}, 14,5^{\circ} \mathrm{C}$ y $13,6^{\circ} \mathrm{C}$, respectivamente (Fig. 2). Al igual que en la temperatura del aire, las temperaturas máximas diarias del agua presentaron la mayor dispersión, con un desvío estándar de $\pm 5,6^{\circ} \mathrm{C}$. Se halló una regresión positiva significativa para las temperaturas mínimas (absolutas) invernales $(\mathrm{p}<0,025$; $\left.R^{2}=0,671\right)$, las mínimas de primavera $\left(p<0,05 ; R^{2}=0,662\right)$ y las mínimas de otoño ( $\left.\mathrm{p}<0,05 ; \mathrm{R}^{2}=0,5\right)$. Los incrementos térmicos fueron $0,8^{\circ} \mathrm{C} / \mathrm{año}$ en las mínimas invernales y $0,7^{\circ} \mathrm{C} /$ año en las mínimas de otoño y primavera. 
Figura 4. Densidad espectral de las temperaturas medias diarias

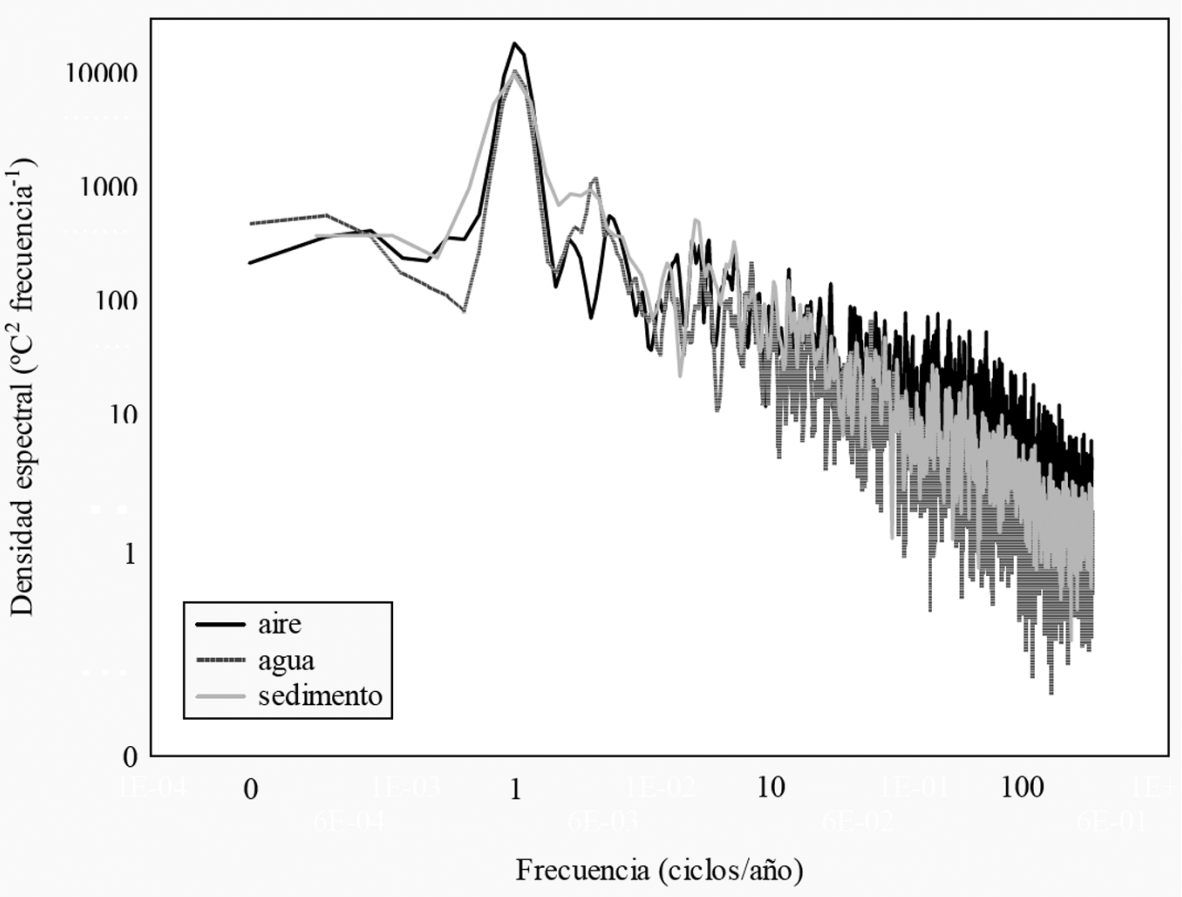

Respecto a las fluctuaciones periódicas de la temperatura del agua, el mayor pico de energía corresponde al ciclo anual (Fig. 4). En tanto, en la escala interanual se observa un pico a los 5,6 años. El ciclo medio anual de temperatura del agua (Fig. 5b) presenta una amplitud térmica de $15,2^{\circ} \mathrm{C}$, oscilando entre $23^{\circ} \mathrm{C}$ a mediados de enero y $7,8^{\circ} \mathrm{C}$ a mediados de julio. Las anomalías calculadas para la temperatura del agua se correlacionaron de forma muy significativa $(a=0,01$; $\mathrm{p}<0,0001)$ con las anomalías en el sedimento $(r=0,62)$ y en el aire $(r=0,49)$ (Fig. 6).

El análisis espectral de la serie de tiempo desestacionalizada (Fig. 7) muestra picos de energía en períodos anuales y semianuales y en ciclos de 85 y 68 días. Respecto a la variabilidad interanual, la energía muestra máximos a 1,6 años y 5,6 años. Las anomalías térmicas filtradas (Fig. 8a) muestran un máximo de $2^{\circ} \mathrm{C}$ a mediados de marzo de 2004. Se observa que las anomalías en el agua siguen la tendencia general de las anomalías en el aire (Fig. 8 y Tabla 1), siendo predominantemente negativas hasta agosto de 2002 (coincidiendo con el evento La Niña 1998/2001) y mayormente positivas a partir de este mes (coincidiendo con los eventos El Niño 2002/03, 2004 y 2006/07) (Fig. 8b). 
Figura 5. Ciclo anual de la temperatura del (a) aire, (b) agua y (c) sedimento en una planicie de marea del estuario de Bahía Blanca. Las líneas negras muestran las fluctuaciones estacionales aproximadas por la ecuación (1) del texto

(a)

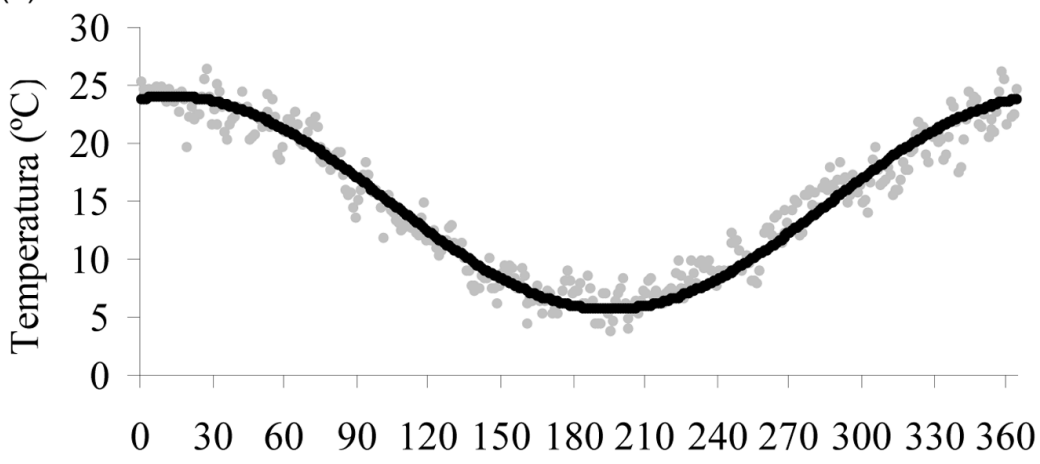

(b)

Día Juliano

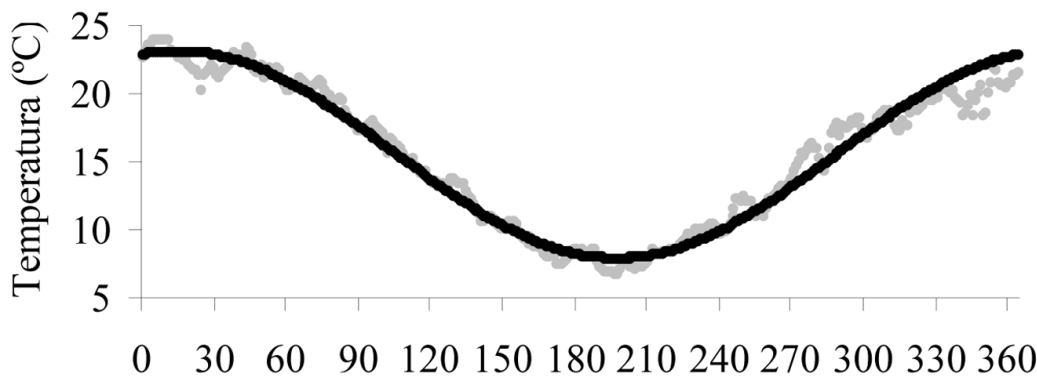

(c)

Día Juliano

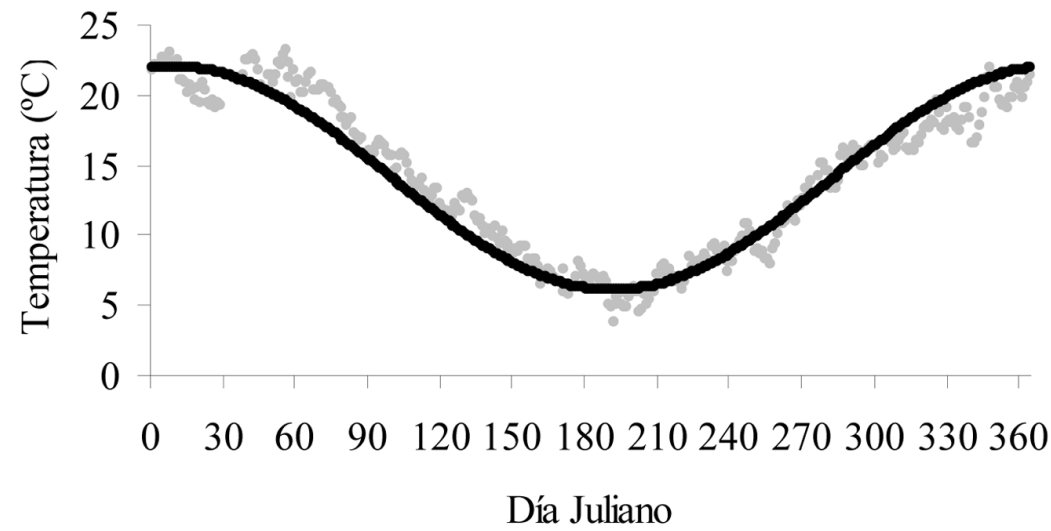


Figura 6. Resultados de una prueba de correlación de Pearson para las anomalías térmicas en el aire, agua y sedimentos de la planicie mareal (período 2000 - 2007)

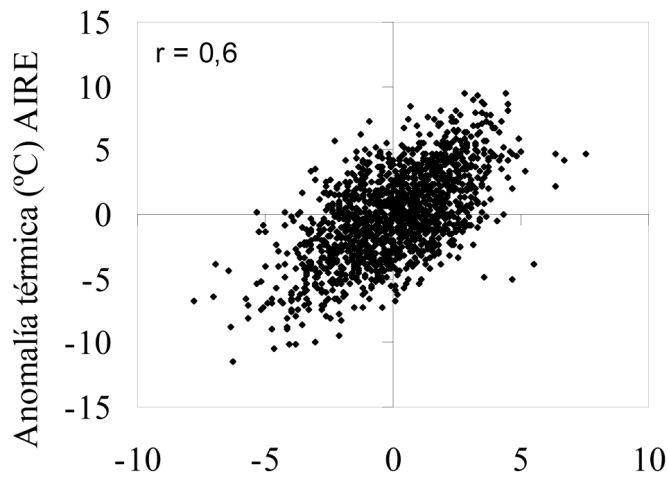

Anomalía térmica $\left({ }^{\circ} \mathrm{C}\right) \mathrm{SED}$

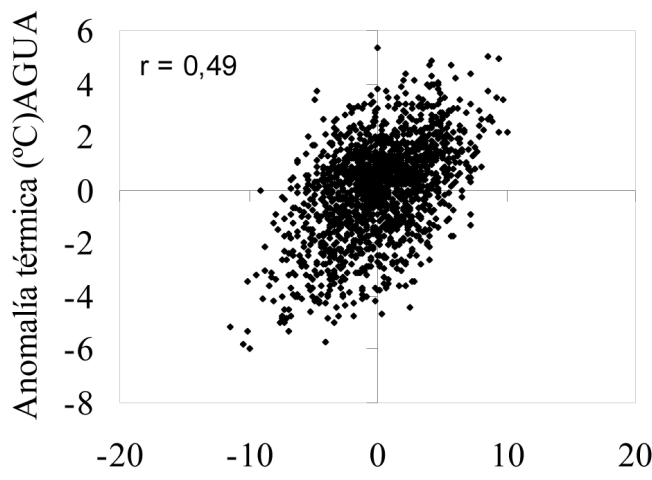

Anomalía térmica $\left({ }^{\circ} \mathrm{C}\right) \mathrm{AIRE}$

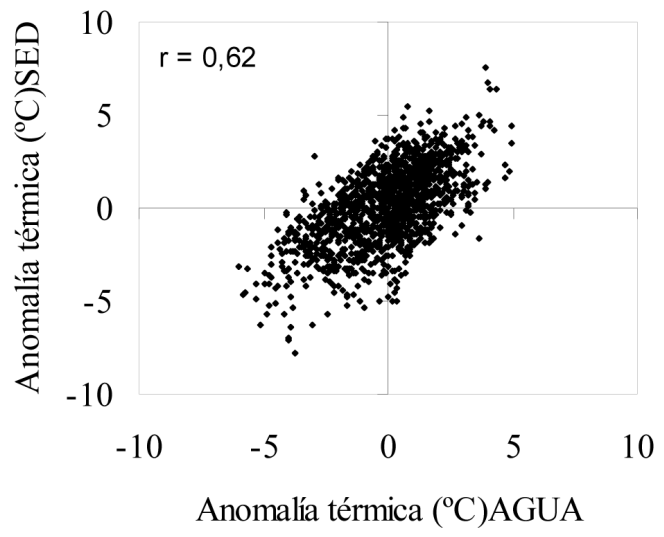

Investigaciones geográficas, $n^{\circ} 48$, pp. 253 - 271 


\subsection{Temperatura del sedimento}

La temperatura del sedimento presentó valores medios de $17,3^{\circ} \mathrm{C}, 13,5^{\circ} \mathrm{C}$ y $10,3^{\circ} \mathrm{C}$ en sus máximas, medias y mínimas diarias, respectivamente (Fig. 2). Nuevamente, las temperaturas máximas diarias mostraron una mayor variabilidad (desvío estándar $= \pm 6,8^{\circ} \mathrm{C}$ ). Se halló una regresión negativa significativa para la temperatura máxima estival $\left(p<0,01 ; R^{2}=0,768\right)$ (Fig. 3b), con una disminución térmica de $0,5^{\circ} \mathrm{C} /$ año. El espectro de energía de la temperatura del sedimento (Fig. 4) muestra una mayor densidad espectral asociada a las señales anuales, siguiendo el comportamiento de la temperatura del aire. Otros máximos importantes presentan frecuencias de 2 ciclos/año y 5 ciclos/año, respectivamente. Para periodicidades interanuales, la temperatura del sedimento muestra un pico de energía a los 3 años.

Figura 7. Análisis espectral de las anomalías térmicas

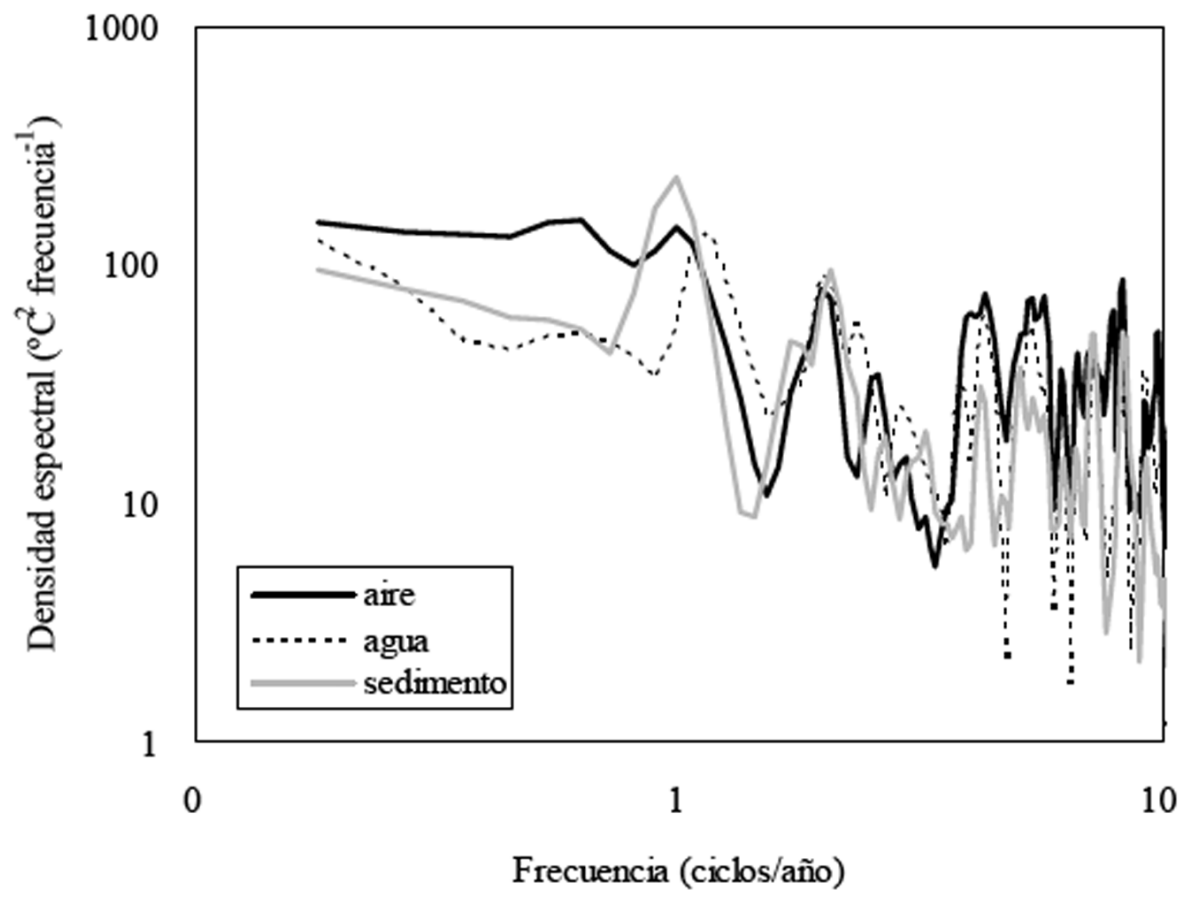

El ciclo medio anual de temperatura del sedimento (Fig. 5c) presenta un valor máximo de $22^{\circ} \mathrm{C}$ a mediados de enero y un mínimo $\left(6^{\circ} \mathrm{C}\right)$ a mediados de julio, con una amplitud térmica de $16^{\circ} \mathrm{C}$. Como se mencionó previamente, las anomalías térmicas en el sedimento mostraron correlaciones muy 
significativas con las anomalías calculadas en el aire y en el agua (Fig. 6). Los espectros de Fourier de dichas anomalías (Fig. 7), al igual que para el caso del aire y del agua, muestran picos de energía en períodos anuales y semianuales. En tanto, en la escala interanual la energía muestra un máximo a los 5,6 años. Las anomalías térmicas filtradas (Fig. 8a) mostraron un máximo de $3,2^{\circ} \mathrm{C}$ a mediados de abril de 2001, coincidente con la máxima anomalía registrada en la temperatura del aire y con la culminación del evento La Niña 1998/2001. Otro máximo positivo $\left(2,1^{\circ} \mathrm{C}\right)$ se observó a principios de abril de 2004, que coincidió aproximadamente con la máxima anomalía registrada en el agua.

Tabla 1. Coeficientes de correlación lineal entre las anomalías térmicas en el estuario (posteriormente al filtrado con medias móviles de 100 días) y el índice del ENOS (IOS)

\begin{tabular}{|c|c|c|c|c|}
\hline & $\mathrm{T}_{\text {aire }}$ & $\mathrm{T}_{\text {agua }}$ & $\mathrm{T}_{\text {sedimento }}$ & IOS \\
\hline $\mathrm{T}_{\text {aire }}$ & 1,00 & $\mathrm{X}$ & $X$ & $X$ \\
\hline $\mathrm{T}_{\text {agua }}$ & $+0,71 *$ & 1,00 & $X$ & $X$ \\
\hline $\mathrm{T}_{\text {sedimento }}$ & $+0,46^{*}$ & $+0,35^{*}$ & 1,00 & $X$ \\
\hline IOS & $-0,11^{*}$ & $-0,27^{*}$ & $-0,1^{*}$ & 1,00 \\
\hline
\end{tabular}

${ }^{*}$ coeficiente altamente significativo ( $p$ menor a 0,0001 )

\section{DISCUSIÓN}

La temperatura media del aire hallada en este trabajo $\left(14,5^{\circ} \mathrm{C}\right)$ es inferior al valor medio anual $\left(15,6^{\circ} \mathrm{C}\right)$ presentado por Piccolo y Diez (2004) para el período 1999 - 2003. Asimismo, la temperatura media del agua para los años $2000-2007\left(14,5^{\circ} \mathrm{C}\right)$ es inferior a los valores medios anuales de $15,2^{\circ} \mathrm{C}$ y $15^{\circ} \mathrm{C}$ hallados por Arango (1985) en Puerto Cuatreros (período 1967 - 1984) y por Piccolo (1987) en Ingeniero White (período 1979 - 1985), respectivamente. Sin embargo, si se observa el comportamiento de la temperatura del aire en la región del estuario de Bahía Blanca durante los últimos 50 años (1951 - 2000), dicho parámetro muestra un aumento paulatino de $0,14^{\circ} \mathrm{C} /$ década en sus valores medios anuales. En particular, en la última década (1991-2000) se registraron incrementos en las temperaturas medias del otoño $\left(+1^{\circ} \mathrm{C}\right)$, invierno $\left(+1,5^{\circ} \mathrm{C}\right)$ y primavera $\left(+0,5^{\circ} \mathrm{C}\right)$, simultáneamente a un descenso de $0.4^{\circ} \mathrm{C}$ en las temperaturas medias estivales respecto a la década anterior (Beigt, 2007). 
Figura 8. (a) Anomalías térmicas en el aire, agua y sedimento durante el período de estudio (se muestran los resultados del filtrado de las anomalías con medias móviles de 100 días). (b) IOS mensual para el período de estudio (Australian Bureau of Meteorology)

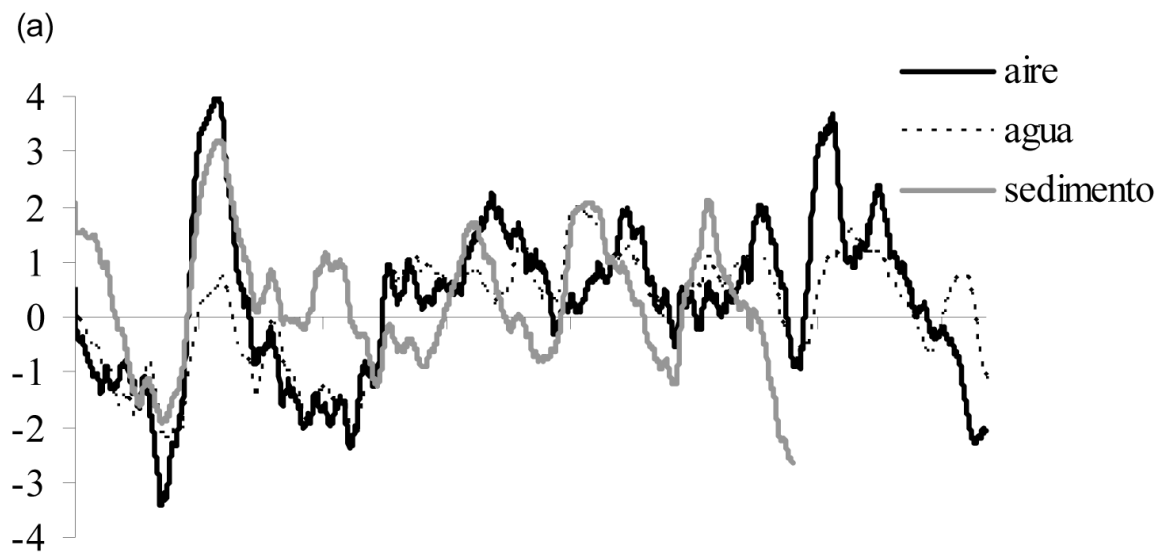

(b)

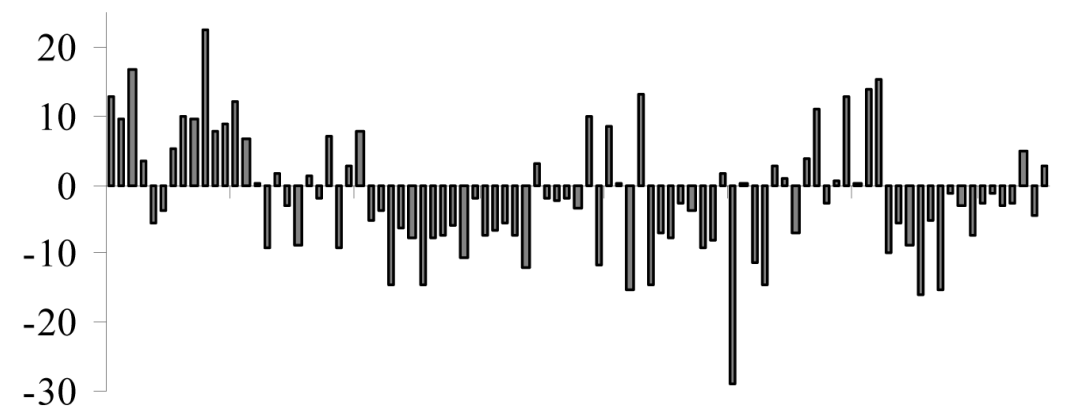

$\begin{array}{llllllll}8 & - & 2 & 0 & 1 & n & 0 & 0 \\ 0 & 0 & 0 & 0 & 0 & 0 & 0 & 1 \\ 1 & 1 & 1 & 1 & 1 & 0 & 0 & 0 \\ 0 & 0 & 0 & 0 & 0 & 0 & 0 & 0 \\ 1 & 1 & 1 & 1 & 1 & 1 & 1 & 1\end{array}$

En este trabajo, el análisis de regresión evidenció una tendencia de incremento en las temperaturas mínimas del aire y del agua en el estuario de Bahía Blanca y de disminución en las temperaturas máximas (estivales) del aire y, por ende, del sedimento. En el agua también se observa una tendencia de disminución en los máximos estivales; sin embargo, ésta no se considera aquí, dada la falta de significación estadística. De ello se deduce que la amplitud térmica se 
ha reducido progresivamente a lo largo del período de estudio. Estos resultados concuerdan con estudios previos (Easterling et al., 1997; Zhai et al., 1999; Griffiths et al., 2005; Limsakul y Goes, 2008), donde se evidencia que los rangos de temperatura se están estrechando en diferentes regiones del mundo, debido a que las temperaturas mínimas se incrementan a una tasa que supera la correspondiente a las temperaturas máximas. Asimismo, los incrementos en las temperaturas mínimas del estuario se condicen con el fenómeno climático señalado por el IPCC (2001c), por el cual, el parámetro más sensible al calentamiento global sería la temperatura mínima.

Naturalmente, el análisis espectral de las temperaturas indicó que el ciclo radiativo anual es la principal fuente de variabilidad térmica en la planicie mareal. Aunque explica el mayor porcentaje de la varianza en los tres medios analizados, la señal se manifiesta más intensamente en la temperatura del aire (Fig. 4). La periodicidad interanual está presente en los tres medios analizados; es de destacar que los resultados del análisis armónico de las temperaturas medias diarias en el estuario de Bahía Blanca se muestran coherentes con el periodograma del IOS presentado por Pasquini y Depetris (2007) para el período 1876 - 2005. Éste presenta una serie de máximos estadísticamente significativos en períodos de 2 a 13 años; en particular, el mayor pico de energía se observa a los 3,6 años, que en este trabajo se evidenció en el aire (3,7 años). Asimismo, el ciclo de 5,6 años observado en la temperatura del agua, aire y sedimento (Fig. 7) se corresponde con el de 5,7 años que presenta el IOS, al igual que el de 1,9 años en el aire es comparable al de 2,3 años del IOS. De esta manera se evidencia que la periodicidad interanual registrada en la temperatura del estuario se halla en estrecha relación con el fenómeno ENOS.

Al comparar las anomalías térmicas con el Índice de Oscilación del Sur (Fig. 8), se observa que la ocurrencia de eventos El Niño durante el período de estudio coincidió aproximadamente con temperaturas del aire y del agua superiores a los valores medios, en tanto que la manifestación de eventos La Niña provocó en el estuario de Bahía Blanca temperaturas del aire y del agua inferiores a las medias. La tabla 1 muestra los coeficientes de correlación lineal entre las anomalías térmicas (filtradas) en el estuario y el Índice de Oscilación del Sur. La temperatura del aire registrada en el estuario mostró una correlación altamente significativa $(a=0,01 ; p<0,0001)$ con el índice del ENOS $(r=-0,11)$. La temperatura del agua a su vez mostró una correlación muy significativa con la temperatura del aire ( $r$ $=0.71)$, la temperatura del sedimento $(r=0,46)$ y el IOS $(r=-0,27)$. En tanto, la temperatura del sedimento mostró correlaciones muy significativas con la temperatura del aire $(r=0,46)$ y el IOS $(r=-0,1)$. Evidentemente, al eliminar las fluctuaciones térmicas de más corto período ( $<100$ días), las temperaturas del 
agua y del aire se muestran más fuertemente correlacionadas entre sí (Figura 6 y Tabla 1). Asimismo, la más fuerte correlación con el IOS se observó en la temperatura del agua, lo cual sugiere que ésta sería la más sensible al forzamiento externo de gran escala.

\section{CONCLUSIONES}

En los últimos 50 años (1951 - 2000), en la región del estuario de Bahía Blanca se ha registrado un aumento de $0,7^{\circ} \mathrm{C}\left(0,14^{\circ} \mathrm{C}\right.$ por década) en las temperaturas medias anuales del aire (Beigt, 2007). En la última década (1991-2000) se registraron incrementos en las temperaturas medias del otoño, invierno y primavera, simultáneamente a un descenso en las temperaturas medias estivales respecto a la década anterior. En este trabajo se evidenció que las temperaturas mínimas en el estuario de Bahía Blanca están registrando incrementos desde el año 2000. La simultánea disminución de las temperaturas máximas estivales determina una reducción progresiva de la amplitud térmica en el estuario, situación que se está registrando en diferentes regiones del mundo.

En relación a las fluctuaciones periódicas de la temperatura en el estuario, se observaron evidencias de periodicidad interanual ( $2-6$ años) en el aire, agua y sedimentos, mostrando un patrón de variabilidad estrechamente relacionado con las periodicidades típicas del fenómeno El Niño (2 - 7 años) (Labat et al., 2005; Pasquini y Depetris, 2007). Si bien estos resultados son preliminares, se demostró que existe una conexión entre los eventos del Pacífico ecuatorial y las anomalías térmicas en el estuario de Bahía Blanca. Específicamente, la ocurrencia de eventos El Niño coincidió con temperaturas del aire y del agua superiores a los valores medios en el estuario. Durante los eventos La Niña, en cambio, se presentaron anomalías térmicas negativas en el aire y el agua.

\section{REFERENCIAS BIBLIOGRÁFICAS}

Ángeles, G. R. (2001): Estudio integrado del estuario de Bahía Blanca. Tesis doctoral. Departamento de Geografía. Universidad Nacional del Sur, Bahía Blanca, 165 pp.

Arango, J. M. (1985): Distribución de parámetros oceanográficos en el estuario de Bahía Blanca. Tesis de licenciatura. Departamento de Física. Universidad Nacional del Sur, Bahía Blanca, 60 pp.

Australian Bureau of Meteorology (2008): S.O.I. (Southern Oscillation Index) Archives-1876 to present. http://www.bom.gov.au/climate/current/soihtml

Beigt, D.; Piccolo, M. C.; Perillo, G. M. E. (2003): «Soil heat exchange in Puerto Cuatreros tidal flats, Argentina», en Ciencias Marinas, no 29 (4B), pp. 595-602. 
Beigt, D. (2007): Balance energético de las planicies de marea del estuario de Bahía Blanca. Tesis doctoral. Departamento de Geografía. Universidad Nacional del Sur, Bahía Blanca, 211 pp.

Capelli de Steffens, A. y Campo de Ferreras, A. (1994): «La transición climática en el SO bonaerense», en SIGEO, n 5, 51 pp.

Easterling, D. R.; Horton, B.; Jones, P. D.; Peterson, T. C.; Karl, T. R.; Parker, D. E.; Salinger, M. J.; Razuvayev, V.; Plummer, N.; Jamason, P. y Folland, C. K. (1997): «Maximum and minimum temperature trends for the globe», en Science, $n^{\circ} 277$, pp. 364-367.

Enfield, D. B.y Mestas-Núnez, A. M. (1999): «Multiscale variabilities in global sea surface temperatures and their relationships with tropospheric climate patterns», en J. Clim., nº12, pp 2719-2733.

GonG, D. Y. y Ho, C. H. (2004): «Intra-seasonal variability of wintertime temperature over East Asia», en Int. J. Climatol., no 24, pp 131-144.

Griffoths, G. M.; Chambers, L. E.; Haylock, M. R.; Manton, M. J.; Nicholls, N.; Baek, H.-J.; Chol, Y.; Della-Marta, P.; Gosai, A.; Iga, N.; Lata R.; Laurent, V.; Maitrepierre, L.; Nakamigawa, H.; Ouprasitwong, N.; Solofa, D.; Tahini, L.; Thuy, D. T.; Tibig, L.; Trewin, B.; Vediapan, K. y Zhai, P. (2005): «Changes in mean temperature as a predictor of extreme temperature change in the AsiaPacific region», en Int. J. Climatol., nº 25, pp 1301-1330.

Houghton, J. T.; Ding, Y.; Griggs, D. J.; Noguer, M.; Van der Linden, P. J.; Dai, X.; Maskell, K. y Johnson, C. A. (2001): Climate Change 2001: the scientific basis. Contribution of Working Group I to the Third Assessment Report of the Intergovernmental Panel on Climate Change. Cambridge University Press.

HurReLl, J. W. (1995): «Decadal trends in the North Atlantic Oscillations: regional temperatures and precipitation», en Science, n 269 , pp 676-679.

HuRReLL, J. W. (1996): «Influence of variations in extratropical teleconnections on northern hemisphere temperature», en Geophys. Res. Lett., n²3, pp 665-668.

Instituto Nacional de Tecnología Agropecuaria (INTA) (2007): el niño 20062007. Nuevo record. http://www.inta.gov.ar/parana/info/documentos/meteorologia/articulos/90716_070620_nino.htm

IPCC (2001a): «Climate change 2001: Impacts, adaptation and vulnerability». En: McCarthy, J.J., Canziani, O.F., Leary, N.A., Dokken, D.J., White, K.S. (Eds.), Contribution of Working Group II to the Third Assessment Report of the Intergovernmental Panel on Climate Change. Cambridge University Press, 1032 pp.

IPCC (2001b): «Climate Change 2001: Synthesis Report». En: Watson, R.T., and the CoreWriting Team (Eds.), A Contribution of Working Groups I, II, and III to the Third Assessment Report of the Intergovernmental Panel on Climate Change. Cambridge University Press, 398 pp. 
IPCC (2001c): Climate Change 2001: the scientific basis - summary for policymakers. Part of the Working Group I Contribution to the Third Assessment Report of the Intergovernmental Panel on Climate Change. Cambridge University Press.

IPCC (2007): Climate Change 2007: the physical science basis - summary for policymakers. Contribution of Working Group I to the Fourth Assessment Report of the Intergovernmental Panel on Climate Change. Cambridge University Press, 18 pp.

Koutsikopoulos, C.; Beillois, P.; Leroy, C. y Taillefer, F. (1998): «Temporal trends and spatial structures of the sea surface temperature in the Bay of Biscay», en Oceanologica Acta, vol. 21, n 2, p.p 335-344.

Labat, D.; Ronchail, J. y GuYot, J. L. (2005): «Recent advances in wavelet analyses: Part 2 - Amazon, Parana, Orinoco and Congo discharges time scale variability», en Journal of Hydrology, no 314, pp. 289-311.

Leathers, D. J.; Yarnal, B. y Palecki, M. A. (1991): «The Pacific/NorthAmerican teleconnection pattern and United States climate: part I regional temperature and precipitation associations», en J. Clim., n ${ }^{\circ}$ 4, pp. 517-528.

Limsakul, A. y Goes, J. I. (2008): «Empirical evidence for interannual and longer period variability in Thailand surface air temperatures», en Atmospheric Research, no 87 , pp. 89-102.

MACKENZIE, B. R. y SCHIEDEK, D. (2007): «Long-term sea surface temperature baselines - time series, spatial covariation and implications for biological processes», en Journal of Marine Systems, nº 68, pp. 405-420.

Masson, D. y Cummins, P. F. (2007): «Temperature trends and interannual variability in the Strait of Georgia, British Columbia», en Continental Shelf Research, no 27, pp. 634-649.

Nezlin, N. P.; Oram, J. J.; Digiacomo, P. M. y Gruber, N. (2004): «Sub-seasonal to interannual variations of sea surface temperature, salinity, oxygen anomaly, and transmissivity in Santa Mónica Bay, California from 1987 to 1997», en Continental Shelf Research, no 24, pp. 1053-1082.

Organización Meteorológica Mundial (OMM) (2006): El Niño/La Niña hoy. http:// www.snet.gob.sv/enos/ENOS20060303

Pasquini, A. I. y Depetris, P. J. (2007): «Discharge trends and flow dynamics of South American rivers draining the southern Atlantic seaboard: An overview», en Journal of Hydrology, no 333, pp. 385-399.

Piccolo, M. C. (1987): Estadística climatológica de Ingeniero White. Período 1980 1985, IADO, Bahía Blanca, 71 pp.

Piccolo, M. C. y Diez, P. G. (2004): «Meteorología del Puerto Coronel Rosales». En: Piccolo, M.C. y Hoffmeyer, M.S. (Eds.), Ecosistema del estuario de Bahía Blanca. IADO. Bahía Blanca, 87-90. 
Piccolo, M. C. y Dávila, P. M. (1991): «El campo térmico de las planicies de marea del estuario de Bahía Blanca», en Actas de las Jornadas Nacionales de Ciencias del Mar, pp 11-15.

Poveda, G.; Waylen, P. R. y Pulwarty, R. S. (2006): «Annual and inter-annual variability of the present climate in northern South America and southern Mesoamerica», en Palaeogeography, Palaeoclimatology, Palaeoecology, no 234, pp. 3-27.

PRESTON, B. L. (2004): «Observed winter warming of the Chesapeake Bay estuary (1949-2002): implications for ecosystem management», en Environmental Management, vol. 34, $\mathrm{n}^{\circ}$ 1, pp 125-139.

Rock, B. N.; Carter, L.; Walker, H.; Bradbury, J., Dingman, S. L. y Federer, C. A. (2002): «Water resources and potential climate change impacts». En: New England Regional Assesment Report (Foundation Document). U.S. Global Change Research Program, 108 pp.

SAlinger, M. J. (2005): «Climate variability and change: past, present and future - an overview», en Clim. Change, $\mathrm{n}^{\circ} 70$, pp 9-29.

Torrence, C. y Compo, G. P. (1998): «A practical guide to wavelet analysis», en Journal of American Meteorological Society, no 79 (1), pp. 61-78.

TREnBerth, K. E. y HuRRelL, J. W. (1994): «Decadal atmosphere-ocean variations in the Pacific», en Clim. Dyn., nº 9, pp. 303-319.

Vargas-Yánez, M.; García, M. J.; Salat, J.; García-Martínez, M. C.; Pascual, J. y MoYA, F. (2007): «Warming trends and decadal variability in the Western Mediterranean shelf», en Global and Planetary Change (en prensa).

Yasunaka, S. y Hanawa, K. (2002): «Regime shifts in the Northern Hemisphere SST field», en J. Meteorol. Soc. Jpn., $\mathrm{n}^{\circ}$ 80, pp. 119-135.

Younes, W. A. N.; Bensoussan, N.; Romano, J-C.; Arlhac, D. y Lafont, M-G. (2003): "Seasonal and interannual variations (1996-2000) of the coastal waters east of the Rhone River mouth as indicated by the SORCOM series», en Oceanologica Acta, no 26, pp. 311-321.

ZHAi, P. M.; Sun, A. y Ren, F. (1999): «Changes of climate extremes in China», en Climatic Change, $\mathrm{n}^{\circ}$ 42, pp. 203-218.

Zhang, Y.; Wallace, J. M. y Battisti, D. S. (1997): «ENSO-like interdecadal variability: 1900-93», en J. Clim., no 10, pp. 1004-1020. 